\title{
Experimental cotton-fibre pulmonary embolism in the rat
}

\author{
BRIAN JOHNSTON, PAUL SMITH, DONALD HEATH \\ From the Department of Pathology, University of Liverpool, Liverpool
}

ABSTRACT Cotton-wool fibres are not uncommonly introduced into systemic veins during medical procedures and are swept into the pulmonary circulation as emboli. To ascertain the histological changes which occur in the pulmonary vasculature, and the time scale of the various cellular reactions we induced experimental cotton-fibre pulmonary embolism in 51 rats. Within an hour the fibres became covered by platelets and plasma proteins and neutrophil polymorphs were attracted around them. By two hours macrophages congregated around the emboli and, with accumulation of epithelioid cells, granulomas were formed. By 16 hours these had increased in size with the appearance of foreign body giant cells, and reached their maximum size in three days. By 16 hours the granulomas were in the wall of the pulmonary artery with rupture of the elastic laminae. At the end of a week granulomas had passed through the wall to reach the alveolar spaces. By the sixth month all were extravascular, lying within the alveolar walls or in the peribronchiolar fibrous tissue. The continuity of the arterial media through which the granuloma had passed was restored without haemorrhage.

Cotton-wool fibres are not uncommonly introduced into systemic veins during medical procedures and are swept into the pulmonary circulation as emboli. We first became aware of this on examination of a lung biopsy specimen from a woman with patent ductus arteriosus and associated pulmonary hypertension which showed a granulomatous reaction in the wall of a muscular pulmonary artery. ${ }^{1}$ This granuloma had formed around a cotton-wool fibre which was probably introduced into the pulmonary circulation during cardiac catheterisation. Contamination of the catheter most likely occurred during its storage in gamgee tissue. Intravenous procedures are commonly carried out at the bedside. When bottles or bags of fluid are changed, joins are made and re-made under less than ideal conditions and cotton fibres from bedding and clothes may contaminate intravenous fluids. The tips of syringe needles may come into contact with cotton-wool fibres either in a sterile container or actually on the skin where fragments may remain after the classical pre-injection procedure of "sterilisation" by swabs of ether or alcohol. Konwaler ${ }^{2}$ described pulmonary emboli of cotton fibres in two men, one with a brain

Address for reprint requests: Professor D Heath, Department of Pathology, University of Liverpool, PO Box 147, Liverpool L69 3BX. abscess and the other with systemic hypertension, both of whom had received intravenous injections. In a study at the Children's Medical Center in Boston, Jaques and Mariscal ${ }^{3}$ found a direct correlation between the amount of intravenous fluid administered and the number of cotton fibres per unit area of lung.

More rarely cotton fibres may be introduced into systemic arteries during angiography. Kay and Wilkins ${ }^{4}$ reported a case in which the mesenteric vessels of a surgically resected segment of ileum were found to be occluded by organising thrombus containing a granulomatous reaction to cotton fibres which had been introduced into the circulation during an aortogram performed 12 days previously. The emboli were thought to have arisen from contamination of the angiographic guide wire or perfusion fluid with cotton fibres. There are many examples of cotton-fibre embolism after carotid or renal arteriography, the source of the fibres being the gauze used to wipe the needle, the guide wire, or the irrigation fluid. ${ }^{5-7}$

The purpose of the present investigation was to determine in detail the histological changes which occur in the pulmonary arteries and arterioles after the impaction into them of cotton-fibre emboli, with particular reference to the time scale of the various cellular reactions. 


\section{Methods}

Absorbent cotton wool was vigorously agitated, with a liquidiser, in sterile normal saline and the suspension of small fibres decanted into a bottle as a stock solution for injecting into rats. The suspension consisted of cotton fibres varying from 3 to $9 \mu \mathrm{m}$ in diameter and 6 to $39 \mu \mathrm{m}$ in length. The concentration of fibres was found to be 500 fibres per ml. Fiftyseven Wistar albino rats (135-191 g) were used in the experiment. They were housed two per cage and given free access to food and water. Fifty-one rats were given an injection of the cotton-fibre suspension and six controls an injection of normal saline. The rats were lightly anaesthetised with ether vapour and one $\mathrm{ml}$ of the suspension injected by a 25 gauge needle into a tail vein dilated by gently warming the rats by placing a lamp over their cage.

The rats were killed with ether vapour after the injection of the cotton-fibres at intervals between one hour and 12 months. Post-mortems were performed immediately. A midline incision was made and the thoracic organs removed intact. The right lung was distended with $10 \%$ buffered formalin and left to fix for two days in the same solution along with the liver, the spleen, and one kidney. In some cases the tail was removed also. Blocks were taken from the superior, middle, and inferior lobes of the right lung. Blocks were taken from the injection sites and decalcified; they were then embedded in paraffin wax. Sections were taken from the lung and stained with haematoxylin and eosin, an elastic-Van Gieson stain, Gomori's aldehyde fuchsin stain, and Martius' scarlet blue stain for fibrin. Sections from other organs were stained with haematoxylin and eosin and an elastic-Van Gieson stain. The heart was dissected in order to weigh independently the left ventricle with interventricular septum, and the right ventricle. The ratio of the former to the latter was calculated to detect right ventricular hypertrophy.

\section{Results}

The overall chain of events was that the cotton-fibre emboli impacted in muscular pulmonary arteries or pulmonary arterioles and then stimulated granulomas to develop around them. The granulomas subsequently migrated through the wall of the vessel concerned to lie in the wall of an alveolus.

IMPACTION OF THE EMBOLI

Cotton fibres are translucent but can be seen readily because they are birefringent. They consist of a cellulose wall with a hollow central core. In the sections from the lungs of two rats killed at up to four hours, all of the cotton-fibre emboli were found to lie inside vessels. Three sections from the lungs of each of these six rats were examined and 100 emboli counted. Ninety-five of the emboli were found in arterioles and the remaining five in muscular pulmonary arteries (fig 1). In 93 instances the emboli

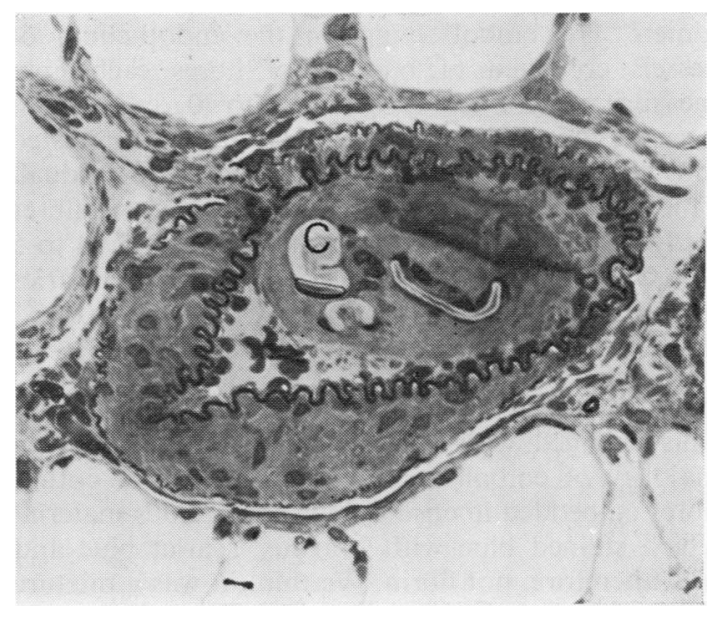

Fig 1 Muscular pulmonary artery 24 hours after injection. Three cotton fibres $(C)$ are in the lumen of the vessel and are embedded in a mass of platelets and plasma protein. The nuclei of monocytes can be seen closely associated with the fibres. Tissue embedded in araldite, sectioned at $2 \mu \mathrm{m}$ thickness. Toluidine blue, $\times 435$.

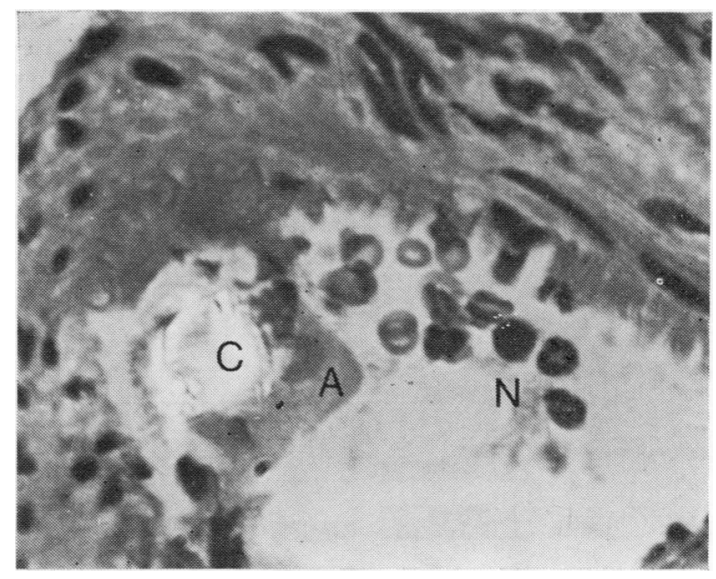

Fig 2 Muscular pulmonary artery four hours after injection. A cotton fibre $(C)$ is closely adherent to the intima of the vessel and is enveloped by amorphous material $(A)$ probably comprising platelets and plasma proteins. There are associated polymorphs $(N)$.

Haematoxylin and eosin, $\times 690$. 
were lying on the endothelium of the patent vessel in which they were situated (fig 2). In five cases the fibres were wedged into an arteriole, blocking it (fig 3), and in two instances they were impaled in the wall of a muscular pulmonary artery. In the latter they were at right angles to the media with part of the fibre stuck into it, the tail projecting into the patent lumen. The emboli lying on the endothelium of vessels consisted of bundles of fibres each fibre measuring 1 to $5 \mu \mathrm{m}$ across $\times 10$ to $30 \mu \mathrm{m}$ in length whereas the fibres wedged in arterioles or impacted into the wall of muscular arteries were individual. The latter fibres tend to be longer. Thus the fibres wedged in pulmonary arterioles were usually 4 to 5 $\times 10$ to $30 \mu \mathrm{m}$ whereas those impaled in arteries measured 4 to $5 \times 30$ to $45 \mu \mathrm{m}$.

\section{CELLULAR REACTION}

This changed with time (fig 4). At one hour the majority of emboli consisted of bundles of cotton fibres embedded in eosinophilic amorphous material which stained blue with Martius' scarlet blue and was, therefore, not fibrin. We think it was a mixture of platelets and plasma proteins for it proved possible to produce the same microscopic appearances by mixing some of the cotton-fibre suspension with fresh blood. Neutrophil polymorphs were adherent to the amorphous material (fig 2) and also penetrated the core of the fibres. This was particularly clear in sections $2 \mu \mathrm{m}$ thick.

By two hours there were fewer polymorphs and less amorphous material around the fibres. Nevertheless, the size of the composite emboli was the same as at one hour due to the accretion of macrophages around and inside the fibres. By four hours polymorphs were sparse and some emboli did not seem to contain any. Some of the fibres were fragmented measuring 1-3 $\times 5-10 \mu \mathrm{m}$ and this may have been the result of the action of the polymorphs and macrophages. By eight hours the amorphous

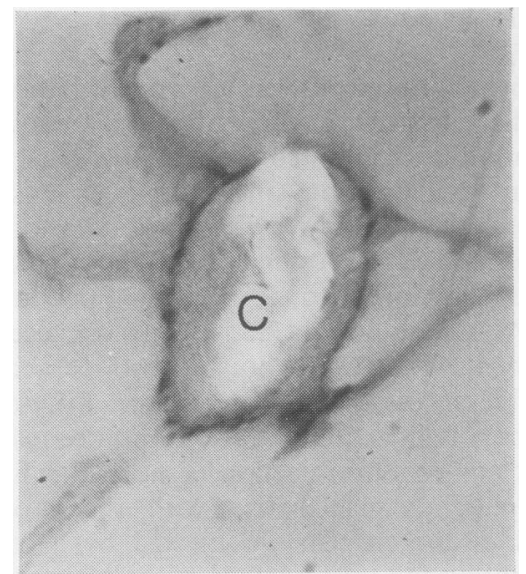

Fig 3 Transverse section of a pulmonary arteriole four hours after injection. The vessel is blocked by a cotton fibre $(C)$ which is also sectioned transversely. The hollow nature of the fibre can be seen. Elastic Van Gieson, $\times 1250$. Partially occluded polarised light.

material had been totally replaced by cells. These were mainly macrophages with scanty cytoplasm. A few macrophages with abundant eosinophilic cytoplasm and indistinct cell boundaries, namely epithelioid cells, began to make their appearance (fig 5). At this stage it had become apparent that the emboli had been converted into granulomas.

At 16 hours the granulomas had increased in size because of the appearance of foreign body type giant cells with abundant eosinophilic cytoplasm and many peripheral nuclei (fig 6). Some of these contained fragments of cotton fibres. There was also an increase in the number of macrophages mainly of the epithelioid variety. At one day there was a further increase in size of the granulomas because of an increase in the number of epithelioid cells. At three days the granulomas reached their maximum size of some $120 \mu \mathrm{m}$ in diameter as a result of an increase
Fig 4 Block diagram to show the material and cells around cotton fibres at progressive periods of time after injection of the fibres. Amorphous material: grade $1=$ present, grade $0=$ absent. Polymorphs, macrophages, epithelioid cells, and giant cells were graded as follows: grade $3=10$ cells or more per embolus per section; grade $2=5-9$ cells per embolus per section; grade $1=1-4$ cells per embolus per section; grade $0=$ none of these cells. The grade of cellular reaction is shown on the ordinate axis and the time interval after injection on the abscissa. 


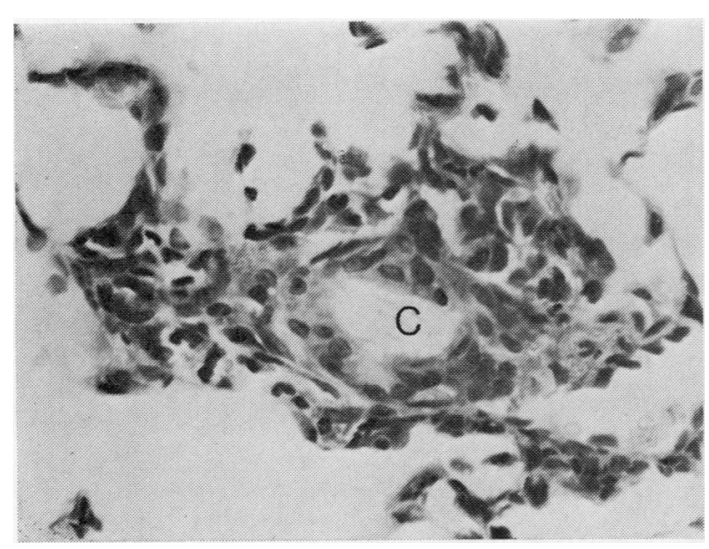

Fig 5 Cotton-fibre granuloma 16 hours after injection. $A$ central cotton fibre $(C)$ is surrounded by a mixture of macrophages and epithelioid cells. $H$ and $E, \times 343$.

in the number of foreign body giant cells (fig 6).

The granulomas then began to decline in size. At

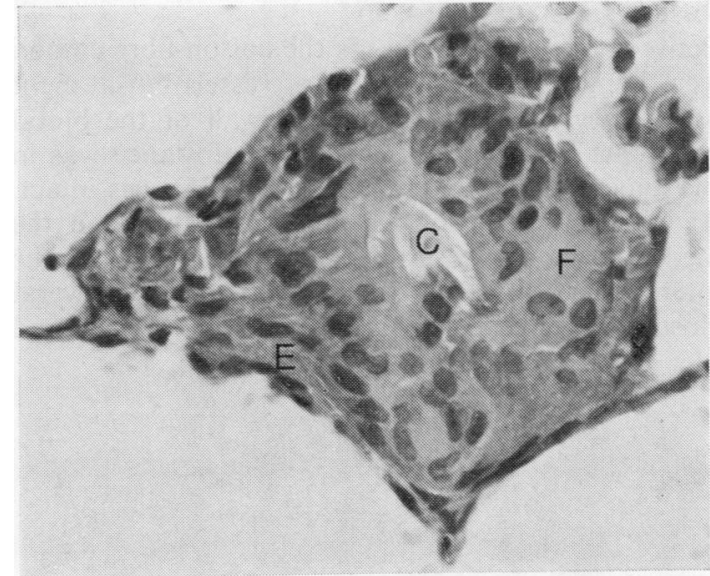

Fig 6 Cotton-fibre granuloma three days after injection. The cotton fibre $(C)$ is entirely surrounded by a large foreign-body giant cell $(F)$ containing many nuclei. This is in turn surrounded by epithelioid cells $(E) . H$ and $E$, $\times 390$.

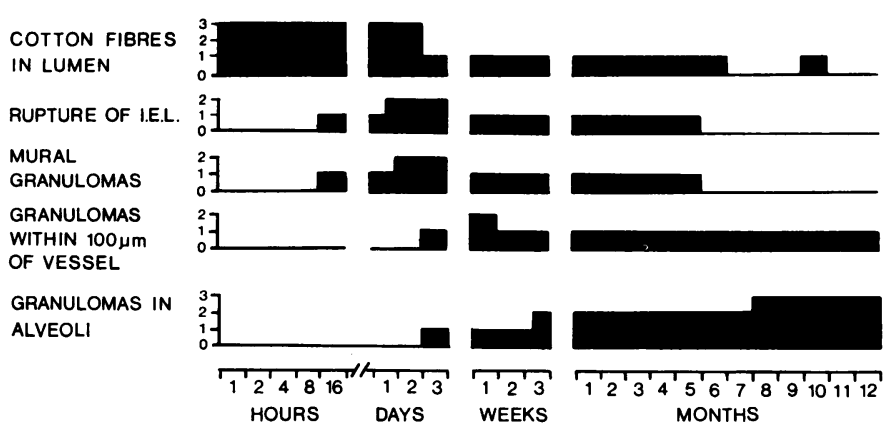

Fig 7 Histological features present at progressive time intervals after injection of the fibres during the process of migration of granulomas through the vessel walls. For each time interval six sections of lung (three per animal) were examined. Three to five cotton fibrecontaining lesions were present in each section and hence for each time interval some 18 to 30 lesions were examined. The incidence of a particular histological feature was graded on the following basis: grade $0=$ none of the embolic lesions showed the feature in question; grade $1=$ up to one-third of lesions showed the feature; grade $2=u p$ to two-thirds of lesions showed the feature; grade $3=$ more than two-thirds of lesions showed the feature. IEL = internal elastic lamina.

one week there was a fall in the number of epithelioid cells with a corresponding augmentation in the number of macrophages. Between one week and one month the cellularity of the granulomas was much the same but by two months only occasional giant cells were seen and there was a reduction in the number of mononuclear cells mainly of the epithelioid variety. As a result there was at this stage a marked decrease in the size of the granulomas. However, between two months and a year the size of the emboli remained fairly constant. They consisted of fragments of cotton fibres surrounded by macrophages, a few being of the epithelioid variety. A few giant cells were usually seen and some contained fragments of cotton fibres.

The fate of the fibres wedged in the pulmonary arterioles was somewhat different. There was a less pronounced cellular reaction round the fibre. Initially this consisted of polymorphs, becoming replaced by macrophages with scanty cytoplasm at about one day. No amorphous material or epithelioid cells were seen. There was no cellular response to the fibres impacted into the walls of the arteries, the tails of the fibres hanging "naked" in the lumen of the vessel. Emboli of this type were not seen after four hours. 
MIGRATION OF GRANULOMAS THROUGH VESSELS WALLS (FIG 7)

Between one and four hours the cotton-fibre emboli were lying within the lumens of vessels but at eight hours they were fused with the wall of the blood vessel. At this stage most of their substance was in the lumen and the internal elastic lamina was intact. At 16 hours some of the granulomas were in the media of the vessel wall itself with rupture of the internal and external elastic laminae at several closely adjacent points (fig 8). Between 16 hours and

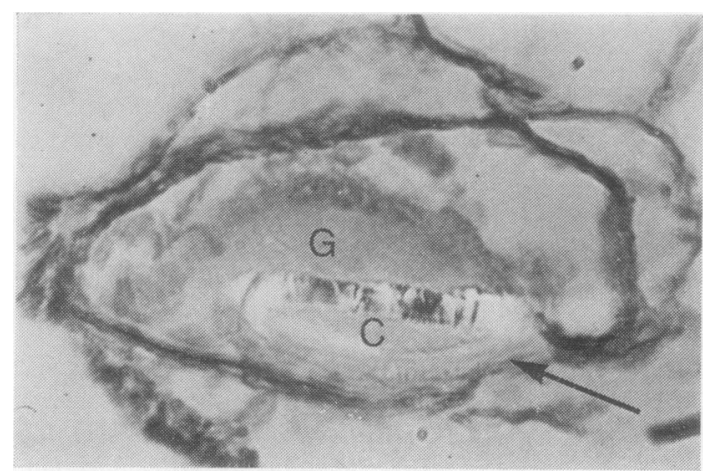

Fig 8 Pulmonary arteriole 16 hours after injection. A hollow cotton fibre $(C)$ is enclosed in a granuloma $(G)$. Where the fibre is in contact with the vessel wall there is a localised rupture of the elastic lamina (arrowed). EVG, $\times$ 665. Partially occluded polarised light.

three days the number of intramural granulomas increased so that by the end of this period most of the granulomas were in vessel walls sometimes extending from their luminal to their adventitial surfaces. A few were in the walls of alveoli next to blood vessels with intact walls. In most cases the defect that contained the granulomas was filled by fibrous tissue, while in some the internal elastic lamina was healed. There was no evidence of haemorrhage from any of the vessels associated with the passage of the granulomas through their walls.

At one week most of the granulomas were situated in the walls of alveoli next to a muscular artery or arteriole (figs 9 and 10) or within the perivascular connective tissue but by three weeks they were found further away from vessels (fig 11). By the sixth month all the granulomas were extravascular, lying either within the alveolar walls or in the peribronchiolar fibrous tissue. Although the elastic laminae had healed, the portal of exit of the granulomas could often be deduced from a ragged appearance of the elastic laminae over part of their circumference. In pulmonary arteries the continuity of the muscular

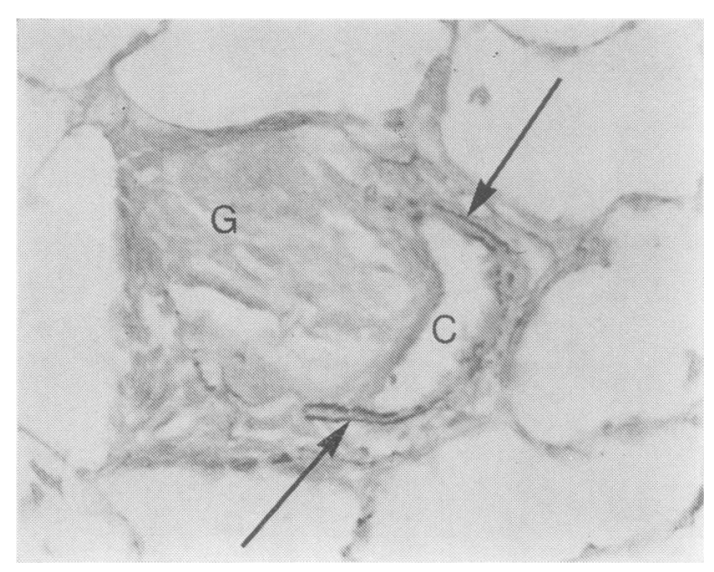

Fig 9 Muscular pulmonary artery one week after injection. The cotton fibres are not visible as they were not photographed using polarised light. They appear as empty spaces $(C)$. The granuloma $(G)$ has ruptured the artery and extended into the surrounding lung parenchyma. The surviving arc of the media, bounded by internal and external elastic laminae, is indicated by arrows. EVG, $\times 370$.

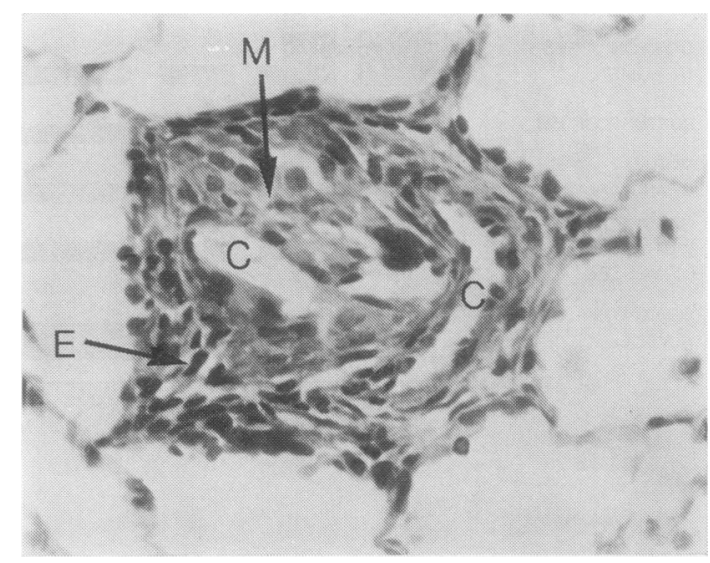

Fig 10 The same lesion illustrated in fig 9 showing cellular detail of the granuloma. The cotton fibres $(C)$ appear as empty slits and are surrounded by macrophages $(M)$ some of which are multinucleated. The periphery of the granuloma consists of epithelioid cells (E). Note the absence of haemorrhage. $H$ and $E$, $\times 370$.

media was restored, if sometimes thinner than normal, and there was no residual scarring. These appearances persisted for the remaining six months.

Fibres were seen impacted end-on in the walls of muscular pulmonary arteries at one, two and fou 


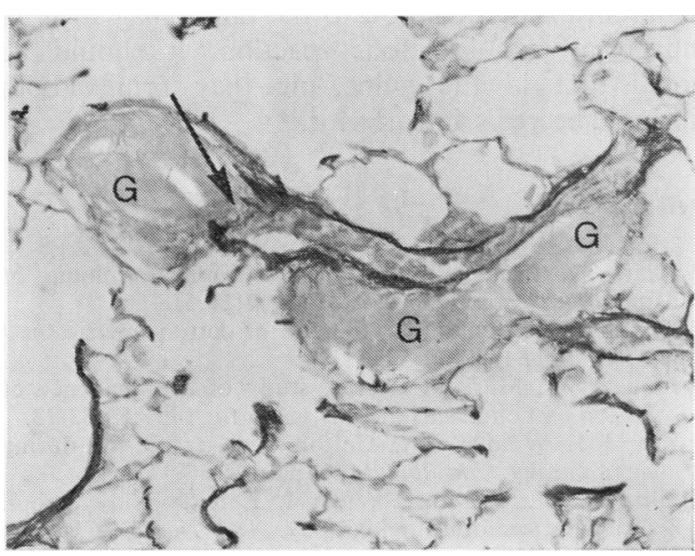

Fig 11 Late stage in the development of granulomas, four weeks after injection. Three granulomas $(G)$ surround an arteriole. The continuity of the elastic lamina has been restored although it has a ragged appearance. At one point (arrowed), the defect has been filled with an irregular mixture of elastin and fibrous tissue. EVG, × 262.

Partially occluded polarised light.

hours. No granuloma formation was seen and it is not clear if or how the fibres penetrated the vessel wall. The fibres may have moved back into the lumen of the vessel to be carried in the flow of blood to impact further down the same vessel or in an arteriole branching off it. There was no evidence of emphysema or hypertensive pulmonary vascular disease.

\section{OTHER ORGANS}

The ratio of the weight of the left ventricle plus interventricular septum to that of the right ventricle was in the range of 3.45-3.91 in the control rats and in the range of $3 \cdot 36-3 \cdot 70$ in the test animals. Sections of the liver, spleen, and left kidney did not contain any cotton-fibre emboli. The suspension of fibres was found to have leaked into the soft tissue at the site of injection into a tail vein. The reaction to this material was similar to that in the lung but the granulomas were larger because of the presence of more cotton fibres.

\section{Discussion}

Our interpretation of the chain of events is in close agreement with the earlier findings of Von Glahn and Hall. ${ }^{8}$ We think that the cotton fibres first become stuck in a mass of platelets and plasma proteins. These conglomerations of fibres and amorphous material became adherent to the endothelium of muscular pulmonary arteries and pulmonary arterioles and it seems likely that this adherence is enhanced by the numerous fine projections from the surface of pulmonary endothelial cells. ${ }^{9}$ Within an hour neutrophil polymorphs accumulate around the fibres but they are soon replaced by macrophages with scanty cytoplasm. These cells predominate during the period of one to 16 hours after impaction of the emboli. They appear to originate as mononuclears which stream into the blood vessel wall from the blood through small defects in the internal elastic lamina which is damaged and ruptured by the fibres. These macrophages mature to form epithelioid cells (eight hours-three days) which fuse to form giant cells over the comparatively long period of 16 hours to three weeks. This accumulation of cells in the media compresses and damages surrounding tissues but does not result in fibrinoid necrosis or acute arteritis. Slowly the cell mass moves through the gap in the elastica and outwards towards the adventitia. The breach in the intima is rapidly covered by growth of endothelium. During the following weeks the granuloma moves outwards, at first assuming a dumb-bell shape and then expanding as it escapes from the compression of the media into the looser peri-arterial tissues. The gap left in the arterial wall by the outward passage of the granuloma is repaired by fibrous tissue with some elastic fibrils. Thus the cotton fibres with surrounding granulomas come to lie in alveolar septa or spaces. Over the period one to twelve months the numbers of giant cells and macrophages fall and the extruded mass comes to consist of fibrous tissue covered by mesothelium. There is no escape of blood since the gap in the medial structures caused by the movement of the granuloma is replaced initially by fibrous tissue but later by smooth muscle cells and repaired elastic laminae to effect some continuity of the muscular media.

The movement of the granulomas through the arterial and arteriolar walls was thought by von Glahn and $\mathrm{Hall}^{8}$ to be in part the result of an increase in pressure inside the vessel. This seems unlikely to us for the normal pulmonary arterial pressure in man is low and as our present findings show there is no evidence that a single injection of cotton fibres leads to pulmonary hypertension or right ventricular hypertrophy. The situation is quite different in such conditions as recurrent embolism from a myxoma of the right atrium for here the repeated and widespread blockage of pulmonary artery by fragments of myxoma may lead to severe pulmonary arterial hypertension which may squeeze the jelly-like material through the media. ${ }^{10}$ In the case of cotton fibres, however, the movement of the granuloma appears to be brought about by accumulations of cells which are derived from the blood stream and raise tissue pressure within the media 
compressing the granuloma outwards.

Cotton-fibre emboli in man produce very similar histological appearances to those which we have seen in the experimental rats. In the lung biopsy specimen from a woman of 49 years with patent ductus arteriosus and pulmonary hypertension referred to above a rod-shaped fibre had impaled a muscular pulmonary artery. Its long axis was radial in relation to the vessel and it had provoked a surrounding granulomatous reaction with macrophages and giant cells, leading to disruption of the internal and external elastic laminae. Thus the appearances were virtually identical to those which we have produced in the rats. It is a matter of speculation as to how many of this patient's pulmonary arteries may have been involved in cottonwool granulomas. It would indeed be a surprising coincidence if a solitary lesion in the lung had been resected in the biopsy; a wide dissemination of fibres seems much more likely. However, we do not think that vascular lesions of this type are of clinical significance. The pulmonary hypertension in the case of this woman was without doubt the result of the altered haemodynamics of the post-tricuspid shunt and not the effect of cotton-fibre emboli. To support this view in the present experimental animals there was no evidence that pulmonary hypertension, manifested as right ventricular hypertrophy, had resulted from this single injection of cotton-fibre emboli.

While our investigations show that in the rat, as in man, cotton-fibre emboli to the pulmonary arteries induce a granulomatous reaction, it should be noted that in the guinea-pig they bring about fibrinoid necrosis and arteritis. ${ }^{11}$

\section{References}

${ }^{1}$ Heath D, MacKinnon J. Cotton-wool granuloma of pulmonary artery. Br Heart $J$ 1962;24:518-20.

${ }^{2}$ Konwaler BE. Pulmonary emboli of cotton fibers. $\mathrm{Am} \mathrm{J}$ Clin Pathol 1950;20:385-9.

${ }^{3}$ Jaques WE, Mariscal GG. A study of the incidence of cotton emboli. Bull Internat A M Mus 1951;32:63-72.

${ }^{4}$ Kay JM, Wilkins RA. Cotton fibre embolism during angiography. Clin Radiol 1969;20:410-13.

${ }^{5}$ Silberman J, Cravioto, Feigin I. Foreign body emboli following cerebral angiography. Arch Neurol (Chicago) 1960;3:711-17.

${ }^{6}$ Chason JL, Landers JW, Swanson RE. Cotton fiber embolism. A frequent complication of cerebral angiography. Neurology (Minneap) 1963;13:558-60.

${ }^{7}$ Adams DF, Olin TB, Kosek J. Cotton fiber embolization during angiography. A clinical and experimental study. Radiology 1965;84:678-81.

${ }^{8}$ Von Glahn WC, Hall JW. The reaction produced in the pulmonary arteries by emboli of cotton fibers. Am J Pathol 1949;25:575-93.

${ }^{9}$ Heath D, Smith P. The pulmonary endothelial cell. Thorax 1979;34:200-8.

${ }^{10}$ Heath D, MacKinnon J. Pulmonary hypertension due to myxoma of the right atrium with special reference to the behaviour of emboli of myxoma in the lung. Am Heart $J$ $1964 ; 68: 227-35$.

${ }^{11}$ Von Glahn WC, Hall JW, Sun SC. Arteritis in guinea-pigs, produced by emboli of cotton, resembling the arteritis of hypersensitivity. Am J Pathol 1954;30:1129-39. 